\title{
Adaptive Multi-Robot Coverage of Curved Surfaces
}

\section{Conference Paper}

Author(s):

Breitenmoser, Andreas; Sommer, Hannes; Siegwart, Roland

Publication date:

2012

Permanent link:

https://doi.org/10.3929/ethz-a-010034947

Rights / license:

In Copyright - Non-Commercial Use Permitted

Originally published in:

Springer Tracts in Advanced Robotics 104, https://doi.org/10.1007/978-3-642-55146-8_1 


\title{
Adaptive Multi-Robot Coverage of Curved Surfaces
}

\author{
Andreas Breitenmoser, Hannes Sommer and Roland Siegwart
}

\begin{abstract}
This paper presents two adaptive coverage algorithms for the deployment of multiple robots into discrete partitions over curved surfaces. The algorithms compute a metric tensor field locally on the surface in order to shape the robots' partitions in position, size, orientation and aspect ratio according to the present anisotropy. The coverage algorithms are further incorporated into a hybrid coverage method for the complete coverage of surface areas. Each robot iteratively deploys and adapts the partition, then subsequently sweeps its assigned area. The algorithms are demonstrated in simulations on different mesh models, including meshes reconstructed from real laser point cloud data.
\end{abstract}

\section{Introduction}

Coverage of work space is an elementary task that arises in multi-robot systems. Multi-robot coverage methods describe how multiple robots coordinate and partition their work load and work space among each other. Depending on the tasks a group of robots has to accomplish, robot coverage can have different meaning: coverage equally involves static and dynamic robot distributions, and notions of sensing as well as actuation. Robot locations in mobile sensor networks for instance are optimized to provide good communication and sensor coverage of the environment $[1,2]$. Similar approaches lead to robot deployments that guarantee fair distribution of work loads among robots, or short response times when providing services to allocated sites [3].

Andreas Breitenmoser, Hannes Sommer and Roland Siegwart

Autonomous Systems Laboratory (ASL), ETH Zurich, Tannenstrasse 3, 8092 Zurich, Switzerland

e-mail: \{andreas.breitenmoser, hannes.sommer\}@mavt.ethz.ch, rsiegwart@ethz.ch 
Another class of coverage tasks requires more permanent movements. In order to continuously monitor an environment, inspect complex structures, or apply tools to a surface in manufacturing, the robots perform coverage by sweeping through their work spaces $[4,5]$.

We are interested in robot coverage of curved surfaces as they are typically found in inspection applications for tanks and tubes of industrial plants. In our work, we consider robots that move directly on the surface $[6,7]$. Spaces in industrial structures are often narrow and thus forbid robots to work together in close side by side formations. Therefore, the robots need to deploy and partition their work space in a reasonable way. However, robot deployment and visual inspection are often not sufficient, and robots are required to sweep their sensor probes in close contact over the surface areas.

Hybrid coverage combines deployment and sweeping motion by performing one after the other [6]. Hybrid coverage methods build on and combine aforementioned coverage concepts. The variant of hybrid coverage we are looking at in this paper first spreads the robots into disjoint regions by constructing a centroidal Voronoi configuration [1]. This is further related to the classical concepts of cell decomposition, just that now the cell decomposition depends on the robot locations rather than on the environment boundaries. Once deployed, each robot sweeps its assigned Voronoi region along a covering path. Here, basically any space filling curve can be applied as long as the kinematic constraints of the robots are met. Examples are swaths similar to those used in the Boustrophedon decomposition on planar and curved surfaces [5].

The main focus of this paper is on the first stage of the two-tired hybrid coverage method. It is important to provide an effective deployment and partitioning of space in the first stage, as each robot needs to cover the entire surface of its assigned Voronoi region in the subsequent second stage. The proposed coverage algorithms make use of anisotropic centroidal Voronoi tessellations [8], and extend our previous work [7] with enhanced adaptivity of the Voronoi regions. The Voronoi regions adapt to local anisotropy, which is defined by a tensor field on the curved surface. The tensor field allows for controlling shape, densitiy and size, as well as orientation and aspect ratio of the Voronoi regions. This new adaptivity may improve multi-robot coverage in several ways. First, adapting the size and orientation of the Voronoi regions according to environment characteristics, such as surface curvature, salient features or representation uncertainty, makes robot movements during operation (e.g., sweeping) in a region on the surface safer and more efficient. Second, adapting the density, orientation or aspect ratio of the Voronoi regions by an input tensor field enables user guidance of the robot configuration. Finally, adapting the shape and size of the Voronoi regions allows to match the region to a sweeping pattern, which is executed by a robot in the region during the second stage of the hybrid coverage method.

Similar to $[7,9,10,11]$, we perform the robot deployment based on a discrete representation and model the environment as a graph. In our previous 
work [7], we suggested two fundamentally different approaches for covering a surface, based on discrete geodesic and approximative Euclidean distance computation. The algorithms in this paper follow up on both approaches in presence of anisotropy. Discrete geodesic alternatives for covering a graph can be found in $[9,10,11]$. Furthermore, geodesic distances under the influence of anisotropy can be computed by Fast Marching methods [12]. The adaptive remeshing of triangular meshes [13], in contrast, applies similar techniques of approximating geodesics by Euclidean distances. The partitioning is driven by an iterative process of pairwise optimization between adjacent regions, which shares similarities with the generation of pairwise optimal partitions as defined in [10]. Anisotropy has been previously used in the context of Voronoi coverage for modeling anisotropic sensors [14]; however, the anisotropy is formulated with respect to the robot positions and not with respect to points in the environment. The concept of adjusting a density function, defined over the areas of the Voronoi regions, is applied in [1] for formation control and robot guidance. Finally, in [3], fat- and skinny-shaped equitable partitions are created using power diagrams, with applications to vehicle routing and optimal workload sharing.

The remainder of the paper is organized as follows. A formal definition of the problem is given in Section 2. Section 3 describes the adaptive coverage algorithms for the deployment of multiple robots on curved surfaces. In Section 4, the hybrid coverage method for area coverage of curved surfaces is presented, which relies on the algorithms of Section 3 in the first stage. Simulation experiments in Section 5 verify the coverage method on synthetic and real data gathered in an inspection scenario. Section 6 concludes the paper.

\section{Problem Definition}

Given a curved connected orientable surface $\mathcal{S} \subset \mathbb{R}^{3}$ with the Riemannian metric induced by the Euclidean scalar product, a group of $n$ robots $r_{i}$, $i \in\{1, \ldots, n\}$, with start positions $\mathbf{q}_{i} \in \mathcal{S}$, is to be distributed over a fair partition $\mathcal{V}=\left\{V_{i}\right\}_{i=1}^{n}$ of $\mathcal{S}$ into regions $V_{i} \subset \mathcal{S}$, each containing one robot at end position $\mathbf{z}_{i} \in V_{i}$. In the partitioning process, it is desirable to adjust the meaning of fair as well as the shapes of the regions $V_{i}$ as much as possible.

First, we drop the constraint of being located on the surface $\mathcal{S}$ and consider the distribution of $n$ points $\mathbf{Z}=\left[\mathbf{z}_{i}\right]_{i=1}^{n} \in \mathbb{R}^{3 n}$ into a partition of a convex three-dimensional submanifold $\Omega \subset \mathbb{R}^{3}$. The resulting simplified problem is typically formulated as an optimization problem, where the following cost functional within the regions $V_{i}$ is minimized,

$$
\underset{\mathbf{Z}, \mathcal{V}}{\operatorname{argmin}} \mathcal{H}_{\text {cont, iso }}(\mathbf{Z}, \mathcal{V})=\underset{\mathbf{Z}, \mathcal{V}}{\operatorname{argmin}} \sum_{i=1}^{n} \int_{V_{i}} \rho(\mathbf{x})\left\|\mathbf{z}_{i}-\mathbf{x}\right\|_{2}^{2} d V(\mathbf{x}),
$$


where we use the integral with volume element $d V$, and $\rho(\mathbf{x})$ describes a non-negative weight (a mass density) at every point $\mathbf{x}$ in $\Omega$. The points $\mathbf{Z}$ and the partition $\mathcal{V}$ are dependent. The partition $\mathcal{V}$ that minimizes the cost functional $\mathcal{H}_{\text {cont, iso }}(\mathbf{Z}, \mathcal{V})$ for fixed points $\mathbf{Z}$ is the Voronoi tessellation. If the points $\mathbf{Z}$ are taken as the generators of the Voronoi tessellation and are moved iteratively to the mass centers of the Voronoi regions, the cost converges to a local minimum and a centroidal Voronoi tessellation (CVT) results.

While choosing $\rho$ gives good control over the fairness of the resulting partition, there is no possibility to influence the regions' shapes.

Therefore, we follow the idea of generalizing the approach given by Equation (1) and replace the Euclidean distance with an anisotropic distance, with which we hope to influence the shapes of the regions,

$$
\underset{\mathbf{Z}, \mathcal{V}}{\operatorname{argmin}} \mathcal{H}_{\text {cont, aniso }}(\mathbf{Z}, \mathcal{V})=\underset{\mathbf{Z}, \mathcal{V}}{\operatorname{argmin}} \sum_{i=1}^{n} \int_{V_{i}} \rho(\mathbf{x}) f\left(d\left(\mathbf{x}, \mathbf{z}_{i}\right)\right) d V(\mathbf{x})
$$

where $f$ is a smooth and strictly increasing function and $d$ denotes a general anisotropic distance metric. Similar to the cost in Equation (1), $\mathcal{H}_{\text {cont, aniso }}(\mathbf{Z}, \mathcal{V})$ is minimized when the points $\mathbf{Z}$ are placed at the anisotropic mass centers of $\mathcal{V}$.

In particular, we consider functions $f(d(\mathbf{x}, \mathbf{z}))=d_{\mathbf{x}, \mathbf{z}}^{p}$ and distances

$$
d_{\mathbf{x}, \mathbf{z}}=\inf _{\substack{\alpha:[0,1] \rightarrow \Omega \\ \alpha(0)=x, \alpha(1)=z}} \int_{0}^{1}\left\|F_{\alpha(t)} \dot{\alpha}(t)\right\|_{p} d t,
$$

where $\|\mathbf{x}\|_{p}=\sqrt[p]{\left|x_{1}\right|^{p}+\left|x_{2}\right|^{p}+\left|x_{3}\right|^{p}}$ denotes the $p$-norm for $p \in \mathbb{N} \backslash\{0\}$ and $F_{\mathbf{x}}$ is a symmetric positive definite matrix smoothly depending on $\mathbf{x} \in \Omega$, which describes the local anisotropy. $F_{\mathbf{x}}$ is called the Finsler tensor field in the following ${ }^{1}$.

We are now interested in formulating the minimization problem of Equation (2) over the surface $\mathcal{S}$. To do so, we follow the same approach as used in [7], and minimize the discrete version of the cost functional defined over the triangle mesh $M$, which is a graph-based representation of the surface $\mathcal{S}$, defined by the graph $G_{M}$. The discretization gives

$$
\mathcal{H}_{\text {disc, aniso }}(\mathbf{Z}, \mathcal{G})=\sum_{i=1}^{n} \sum_{v \in G_{i}} \int_{A_{v}} \rho(\mathbf{x}) d_{\mathbf{x}, \mathbf{z}_{i}}^{p} d S(\mathbf{x}),
$$

where $d S$ denotes the surface element. The partition is represented by $\mathcal{G}=$ $\left\{G_{i}\right\}_{i=1}^{n}$, with the subgraphs $G_{i} \subset G_{M}$ given by sets of connected vertices $v$. $A_{v}$ denotes the area of the dual cell of each vertex. In the case of a planar

\footnotetext{
1 This yields the usual distance of the Finsler manifold, given by $\Omega$ together with the Finsler function $(\mathbf{x}, \mathbf{v}) \mapsto\left\|F_{\mathbf{x}} \mathbf{v}\right\|_{p}$.
} 
mesh, this corresponds to the Voronoi region around $v$ obtained from the Voronoi diagram with the mesh vertices as generators.

To determine the distance $d_{\mathbf{x}, \mathbf{z}}$, the integral in Equation (3) is approximated with a sum of lengths $\left\|\widetilde{F}_{\mathbf{x}_{l}}\left(\mathbf{x}_{l+1}-\mathbf{x}_{l}\right)\right\|_{p}$ of segments given by a sequence of nodes $\mathbf{x}_{l}, l \in \mathbb{N}$, in the graph $G_{M}$, connecting $\mathbf{x}$ and $\mathbf{z}$.

Although $\mathcal{S}$ is a two-dimensional manifold, it is not sufficient in the discrete version to define $F_{\mathbf{x}}$ on the two-dimensional tangent spaces of $S$ because the distance vectors $\left(\mathbf{x}_{l+1}-\mathbf{x}_{l}\right)$ are not restricted to the tangent spaces of $\mathcal{S}$ at $\mathbf{x}_{l}$. So, however we choose $F_{\mathbf{x}}$ on the tangent spaces, it must be given as a three-by-three matrix $\widetilde{F}_{\mathbf{x}}$. This matrix can be constructed from the desired matrix $F_{\mathbf{x}}$ given in a basis of $T_{\mathbf{x}} \mathcal{S}$, e.g., such that in the local basis extended

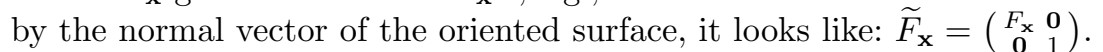

In the following, we pursue two different approaches of choosing the sequence of nodes $\mathbf{x}_{l}$ for the distance computation. In the first approach, $d_{\mathbf{x}, \mathbf{z}}$ is computed for arbitrary $p$-norms as the discrete geodesic distance. The distance segments correspond to the edges along a shortest path from $\mathbf{x}$ to $\mathbf{z}$ on the graph $G_{M}$. In the second approach, the path distance $d_{\mathbf{x}, \mathbf{z}}$ is approximated by the length of a single segment, which connects $\mathbf{x}$ and $\mathbf{z}$ along the direct shortcut through $\mathbb{R}^{3}$, using the 2 -norm. We arrive this way at the directional distance as defined in $[8]^{2}$. That yields $d_{\mathbf{x}, \mathbf{z}} \simeq\left\|\widetilde{F}_{\mathbf{x}}(\mathbf{z}-\mathbf{x})\right\|_{2}=$ $\sqrt{(\mathbf{z}-\mathbf{x})^{T} \widetilde{K}_{\mathbf{x}}(\mathbf{z}-\mathbf{x})}$, where $\widetilde{K}_{\mathbf{x}}:=\widetilde{F}_{\mathbf{x}}^{T} \widetilde{F}_{\mathbf{x}}$, which is a usual anisotropic quadratic distance in $\mathbb{R}^{3}$.

For the second approach, under the approximation that $\widetilde{K}_{\mathbf{x}_{v}}$ (in short $\widetilde{K}_{v}$ ) is constant over a given vertex area $A_{v}$, the cost functional $\mathcal{H}_{\text {disc, aniso }}$ can be rewritten with $d_{\mathbf{x}, \mathbf{z}_{i}}^{2}=\left(\mathbf{z}_{i}-\mathbf{x}\right)^{T} \widetilde{K}_{\mathbf{x}}\left(\mathbf{z}_{i}-\mathbf{x}\right)$ as

$$
\begin{gathered}
\mathcal{H}_{\text {disc, aniso }}(\mathbf{Z}, \mathcal{G})=\sum_{i=1}^{n} \sum_{v \in G_{i}}\left[W_{v}+\left(\mathbf{z}_{i}-\gamma_{v}\right)^{T} \widetilde{K}_{v}^{*}\left(\mathbf{z}_{i}-\gamma_{v}\right)\right]= \\
\sum_{i=1}^{n}\left[\sum_{v \in G_{i}} W_{v}+\sum_{v \in G_{i}}\left(\gamma_{v}^{T} \widetilde{K}_{v}^{*} \gamma_{v}\right)+\mathbf{z}_{i}^{T}\left(\sum_{v \in G_{i}} \widetilde{K}_{v}^{*}\right) \mathbf{z}_{i}-2 \mathbf{z}_{i}^{T}\left(\sum_{v \in G_{i}} \widetilde{K}_{v}^{*} \gamma_{v}\right)\right],
\end{gathered}
$$

where $\gamma_{v}$ is the centroid of $A_{v}, W_{v}=\int_{A_{v}} \rho(\mathbf{x})\left(\gamma_{v}-\mathbf{x}\right)^{T} \widetilde{K}_{\mathbf{x}}\left(\gamma_{v}-\mathbf{x}\right) d S(\mathbf{x})$, and $\widetilde{K}_{v}^{*}=m_{v} \widetilde{K}_{v}$, with $m_{v}=\int_{A_{v}} \rho(\mathbf{x}) d S(\mathbf{x})$, the concentrated weight over vertex area $A_{v}$. From Equation (5) follows that it is sufficient to minimize for the last two terms, since the first two terms do not depend on a particular choice of $\{\mathbf{Z}, \mathcal{G}\}$. The locations of the points $\mathbf{Z}$ can be chosen freely. However,

2 Note that this distance measure is not symmetric and thus no proper distance metric as such; however, it is consistent with the definition of the standard Voronoi diagram in the isotropic metric and straightforward to compute. See [8] for further discussions. 
if we select the generators $\mathbf{Z}$ in Equation (5) to be the mass centers of the Voronoi regions, which is in accordance with $[1,8]$ and the notion of CVT, the last two terms in Equation (5) can be further simplified. The anisotropic mass centers are obtained as $\mathbf{z}_{i}=\operatorname{inv}\left(\sum_{v \in G_{i}} \widetilde{K}_{v}^{*}\right) \sum_{v \in G_{i}} \widetilde{K}_{v}^{*} \gamma_{v}$, which are the minimizers of the cost functional in Equation (4). Substituting $\mathbf{z}_{i}$ for the last two terms of Equation (5) leads to the partial cost

$\mathcal{H}_{\text {disc, aniso }}^{*}(\mathbf{Z}, \mathcal{G})=\sum_{i=1}^{n}\left[-\left(\sum_{v \in G_{i}} \widetilde{K}_{v}^{*} \gamma_{v}\right)^{T} \operatorname{inv}\left(\sum_{v \in G_{i}} \widetilde{K}_{v}^{*}\right)\left(\sum_{v \in G_{i}} \widetilde{K}_{v}^{*} \gamma_{v}\right)\right]$,

which does not depend on the generators $\mathbf{z}_{i}$ anymore. In the isotropic case, Equation (6) becomes

$$
\mathcal{H}_{\text {disc }, \text { iso }}^{*}(\mathbf{Z}, \mathcal{G})=\sum_{i=1}^{n}\left[-\frac{\left\|\sum_{v \in G_{i}} m_{v} \gamma_{v}\right\|_{2}^{2}}{\sum_{v \in G_{i}} m_{v}}\right] .
$$

The original problem of distributing $n$ robots $r_{i}$ over the surface $S$ can now be solved for the second approach by simply minimizing Equation (6), or Equation (7) respectively, and letting $\mathbf{Q}=\left[\mathbf{q}_{i}\right]_{i=1}^{n}$ approach $\mathbf{Z}=\left[\mathbf{z}_{i}\right]_{i=1}^{n}$ over time. The partial cost $\mathcal{H}_{\text {disc, aniso }}^{*}(\mathbf{Z}, \mathcal{G})$ and $\mathcal{H}_{\text {disc, iso }}^{*}(\mathbf{Z}, \mathcal{G})$ are minimized in an efficient way by exchanging vertices between neighboring Voronoi regions, which only requires updates of the sums $\sum \widetilde{K}_{v}^{*} \gamma_{v}$ and $\sum \widetilde{K}_{v}^{*}$, or $\sum m_{v} \gamma_{v}$ and $\sum m_{v}$ respectively.

For the first approach, the original cost in Equation (4) must be minimized; gradient descent methods provide a practical solution $[1,7,9]$. This involves finding the direction of fastest decline of distance. We use the negated tangent vector of the minimizing geodesic, which differs from its gradient $[9]^{3}$.

The Finsler tensor given by $F_{\mathbf{x}}$ offers several ways to adapt the partition over the surface $\mathcal{S}$ (see Figure 1). The orientation of the Voronoi regions is influenced by the directions of the eigenvectors of $F_{\mathbf{x}}$. The regions' aspect ratio is given by the ratios of the eigenvalues of $F_{\mathbf{x}}$, measuring the strength of directionality. As mentioned at the beginning of the section, the regions' size can be changed by the weighting factor or mass density $\rho$. In addition, the distance in the cost functionals can assume the general $p$-norm. Depending on $p$, the $p$-norm results in a more circular or square-shaped distance field, which leads to modifications in the shapes of the regions.

Here, a remark is in place: the $p$-norm only applies to the first approach, which computes discrete geodesic distances. Note that the second approach with approximate distance computation, as presented in here, requires $p=2$. This is inherent to above derivation (from Equation (4) to Equation (5)),

3 In fact, they even differ in their mathematical type (tangent vector vs. tangent covector). Their value's relation can be found for $p=2$ in [9]. This must be generalized for $p \neq 2$. However, this is not included here for the sake of brevity. 

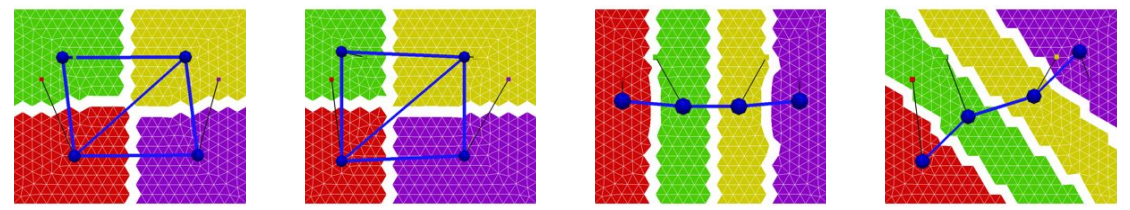

Fig. 1 Adaptive deployment of four robots with uniform, isotropic (to the left) and anisotropic (upward and diagonal) metric tensor fields provided by the user guidance.

which is based on the parallel axis theorem. The parallel axis theorem as such, however, relies on specific properties of the $2-$ norm.

\section{Adaptive Surface Coverage}

The iterative construction of a Voronoi tessellation on a graph $G_{M}$, representing a triangle mesh $M$, forms the basis for our adaptive surface coverage algorithms. Each Voronoi region is owned by a robot $r_{i}$ and represented by a subgraph $G_{i} \subset G_{M}$. As the partitioning process evolves, the Voronoi regions - and together with the regions the robots - are distributed over the surface. The subgraphs $G_{i}$ result for the first approach from minimizing the cost with discrete geodesic distance in Equation (4), and for the second approach from minimizing the cost with approximative Euclidean distance in Equation (6) or Equation (7). The basic algorithms have been introduced in [7] and are restated in the following. Algorithm 1 summarizes the approaches' main function, and Algorithm 2 and Algorithm 3 describe the coordination functions of the two approaches.

The first approach computes a discrete geodesic distance by propagating a wavefront over the graph. Each robot computes a distance field from its current position $\mathbf{v}_{\mathbf{p}_{i}}$, which is the robot's position $\mathbf{p}_{i} \in \mathcal{S}$ projected onto the graph $G_{M}$, to positions of neighboring robots on the graph. As soon as the wavefront reaches a neighbor's vertex, a wavefront is propagated back in order to find the boundary vertices with equal discrete geodesic distance between the two robots. This procedure is continued by each robot $r_{i}$ until the complete Voronoi partition $\mathcal{G}$ is constructed. Once the Voronoi regions are computed, the robots move toward the regions' mass centers. The regions are updated continuously, and it is sufficent to determine the direction toward the mass center instead of its absolute position. As the direction, we use the negated tangent vector of the minimizing geodesic (see Section 2).

The second approach minimizes the cost by exchanging boundary vertices iteratively across the boundaries of adjacent regions. For each vertex $v_{A} \in G_{i}$ located at a region boundary, following local vertex exchanges are possible. If a vertex at the boundary next to $v_{A}$ has not yet been assigned to any 

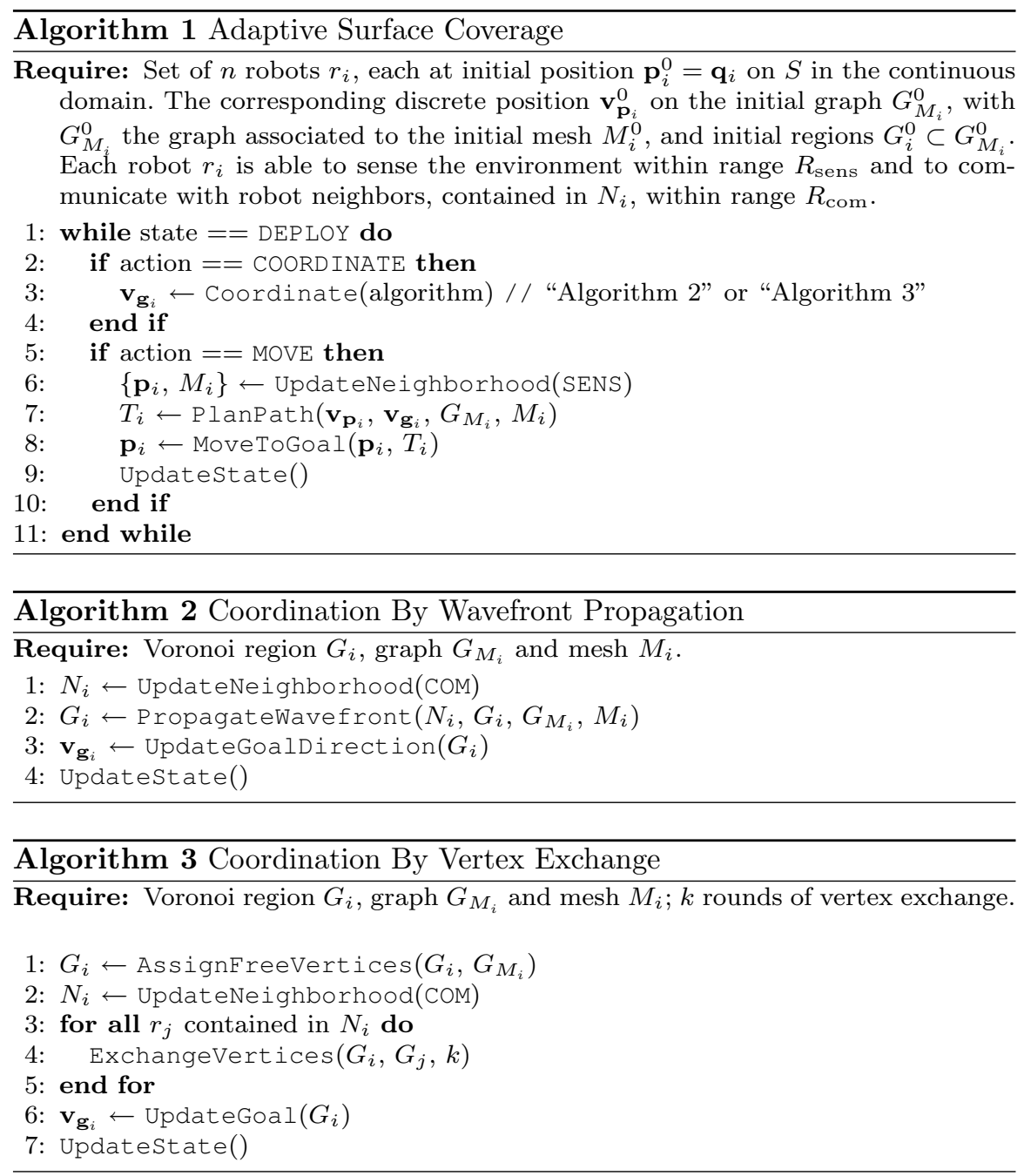

region, it is directly added to $G_{i}$. If there exists at least one neighbor vertex $v_{B} \in G_{j}, G_{i} \cap G_{j}=\emptyset$, the change in the cost of Equation (6) or Equation (7) is evaluated for the following three cases: 1) the vertex $v_{A}$ is added to $G_{j}$, 2) the vertex $v_{B}$ is added to $G_{i}$, or 3) no vertex is exchanged across the boundary. The case resulting in the highest reduction of cost is used for the local update of the two Voronoi regions. If a vertex exchange leads to a disconnected region, the last case applies and the respective vertex will not be exchanged. Note that the last case does not affect the convergence of the overall algorithm; the cost is not minimized but remains unchanged in this iteration step. However, over subsequent iterations the pairwise optimization by exchanging vertices among adjacent regions minimizes the overall cost. 
Each robot is assumed to be equipped with communication and sensing devices. The information collected from the environment is stored in the triangle mesh maps $M_{i} \subset M$, and each mesh map is associated with a graph $G_{M_{i}} \subset G_{M}$. For applications, where the algorithms are required to adapt to characteristics of the surface (e.g., surface curvature), the matrix $\widetilde{F}_{\mathbf{x}}$ is computed from the mesh map $M_{i}$. A Voronoi region is defined as the subgraph $G_{i} \subset G_{M_{i}}$. The neighborhood $N_{i}$ of a robot $r_{i}$ includes all the neighboring robots $r_{j}$ within communication range $R_{\text {com }}$, together with the additional information of their positions, Voronoi regions and mesh maps. The UpdateNeighborhood function updates $N_{i}$; for argument SENS, the robot $r_{i}$ updates its own position and mesh map, for argument COM, the robot detects other robots and updates its mesh map from communicated information. If the meshes of two neighboring robots overlap, the meshes are merged, $M_{i} \leftarrow M_{i} \cup M_{j}, M_{j} \leftarrow M_{i} \cup M_{j}$. A matching among two neighboring robots is only established if the robots' Voronoi regions are adjacent, i.e., if two vertices in $G_{i}$ and $G_{j}$ are adjacent in the graph $G_{M}$.

The adaptive surface coverage algorithms are divided into a coordination and a moving action. The coordination action implements the previously described cost minimization process. In the coordination action of the second approach, $k$ rounds of vertex exchange are performed by the ExchangeVertices function. With $k$ chosen large enough, a robot will not enter a local vertex exchange with another robot neighbor before a partition with optimal cost between its own and the current neighboring region is obtained. Provided that a vertex exchange procedure can find the global optimum for the two regions, this leads to pairwise optimal partitions similar to [10]. The coordination action updates the goal direction and goal position $\mathbf{v}_{\mathbf{g}_{i}}$ on the graph for each region and robot, which is then handed over to the moving action of the algorithms in order to deploy the robots on the surface. During the moving action the robots navigate on the surface. Each robot perceives the environment and computes the shortest path to its current goal. The PlanPath function updates the path $T_{i}$, based on the discrete positions $\mathbf{v}_{\mathbf{p}_{i}}$, whereas the MoveToGoal function controls the robot along the path.

In the Voronoi coverage method of [1], the robots act as the generators and converge to the mass centers of their Voronoi regions. However, this is no longer a necessary condition in our case, as the Voronoi regions are generated either by wavefront propagation between vertices or by local vertex exchanges of boundary vertices on the underlying graphs. The coordination and moving actions are loosely coupled ${ }^{4}$. The decoupling of the two actions allows to place the robots' goal positions at different locations that may improve the overall performance of the algorithms, with benefits such as lower cost paths, better accessibility of the goal positions, or better sensor coverage. Placing

\footnotetext{
4 The dependence is only given through the communication and the environment update; the robot positions influence what the robots will communicate, perceive from their environment and incorporate into the mesh maps.
} 
goals closer to the boundary of a Voronoi region, for example, could increase the explorative behavior of the basic coverage algorithms.

\section{Application to Hybrid Coverage Control}

This section explains how the adaptive surface coverage algorithms can be applied within the hybrid coverage framework to achieve area coverage on a surface. The hybrid area coverage method is outlined in Figure 2 and Algorithm 4 . The idea behind hybrid coverage is to combine robot deployment and sweeping motions [6]. The method starts with the robots spreading out on the surface. The robots cooperatively partition the surface and get their assigned areas of operation. In this first stage, the adaptive surface coverage algorithms from Section 3 are applied to realize an effective deployment and adaptive decomposition of the surface. The provided adaptivity can be actively used to shape the Voronoi regions and simplify the coverage of the area, e.g., leading to compact or more elongated shapes that support circular spiralling or rectangular back-and-forth sweeping patterns. Upon convergence, the robots switch to the second stage, where each robot sweeps its assigned region locally to search the area. Depending on the size of the environment to be covered and the range of coverage of the single robots, the two stages of deployment and sweeping (state DEPLOY and SWEEP in Algorithm 4) are iterated. The robots relocate and redistribute outside the already covered area by applying the hybrid coverage subroutines AdaptiveSurfaceCoverage and SweepSurfaceCoverage again. By iterating the process, the complete surface is finally covered by the robots.

Once a Voronoi region is covered, it is marked as covered in the robot's mesh map $M_{i}$ and is locked; the robot communicates the status to its neighbors, which update their mesh maps accordingly. As the adaptive surface coverage algorithms deploy the robots by generating a Voronoi tessellation, a dual Delaunay graph is established simultaneously (see Figures $1-4$ ). The graph connects the Voronoi regions, represents the surface topology and gives a simplified low resolution representation of the environment. This representation remains valid and may serve as roadmap for future relocation and redistribution phases of the robots. The covered regions are known to the robots as they have swept and explored these areas before. Hence, paths transferring from one location on the surface to another are preferably planned through the known and safe area of the regions along the Delaunay graph.

Besides covering and locking of regions, many more operations on the regions are possible. A robot can leave its region uncovered and reassign it to other robots for coverage, or ask other robots for support. Regions can be deleted cooperatively if a robot fails or relocates. Furthermore, a new region is instantiated whenever a robot joins during deployment, or a region is created inside already covered area in order to initiate redundant coverage. 

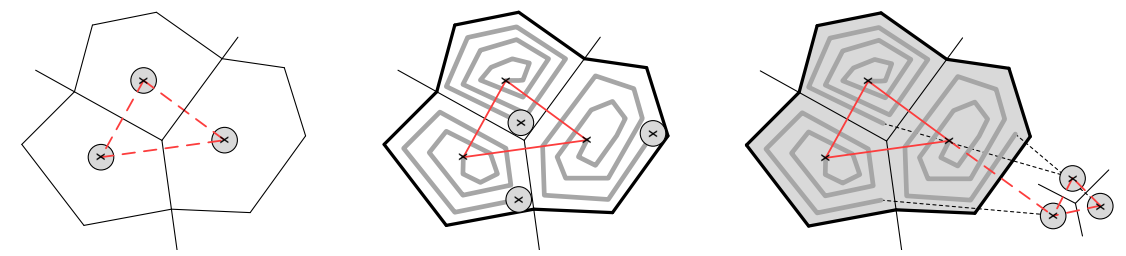

Fig. 2 Hybrid area coverage. Left: Robot deployment. Center: Sweeping motions. Right: Relocation and redistribution. The dual Delaunay graph is marked in red.

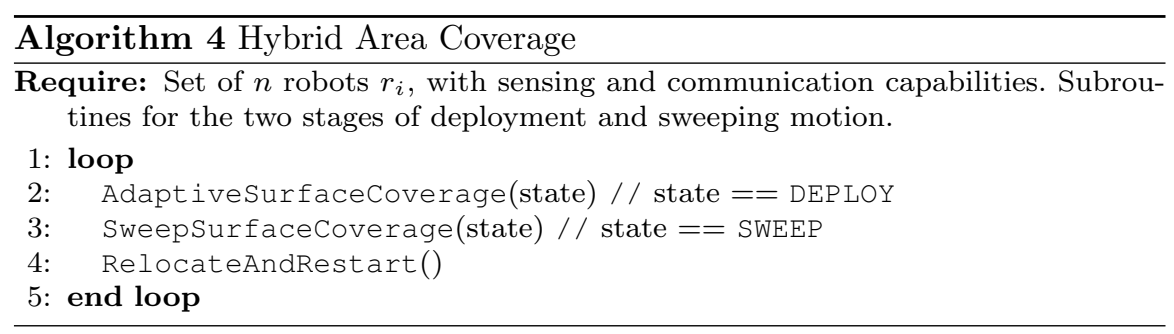

\section{Simulation Experiments}

The adaptive surface coverage and the hybrid area coverage algorithms are tested on different surfaces. The surface models vary in complexity and consist of synthetic meshes, and meshes obtained by surface reconstruction from real laser point clouds. In the following simulations we assumed that the robots always remain connected (i.e., $R_{\text {com }}=\infty$ ).

The first experiment demonstrates the adaptivity of the Voronoi coverage. Figure 1 shows how four robots are deployed on a planar mesh under varying user guidance. The metric tensor field is specified directly by the user. The first deployment is uniform, which sets equal weights to all vertices and directions. The second deployment is isotropic and directs the robots to the left of the mesh. The metric tensor field for the first two deployments is of the form $\widetilde{K}_{v}^{*}=m_{v} I_{3}$, where $m_{v}$ is constant for the uniform deployment and varies as function of the location for the isotropic deployment. The third and fourth deployments use an anisotropic metric. The Voronoi partitions point along the principal directions of $90^{\circ}$ upwards and $45^{\circ}$ to the left.

In the second and third experiment, 10 and 50 robots are distributed on the synthetic mesh model of a torus (Figure 3) and a cactus (Figure 4). The deployment on the torus is isotropic and the deployment on the cactus is anisotropic. In both cases, the matrix $\widetilde{F}_{v}$ is constructed from the local curvature of the surface, given by weights $\rho(\mathbf{v})=1+\sqrt{k_{v, 1}^{2}+k_{v, 2}^{2}}$, the principal curvatures $k_{v, 1}$ and $k_{v, 2}$, and the principal directions. The principal curvatures and principal directions are estimated from the mesh model following [15], and are shown in Figure 5 on the left and in the center. 

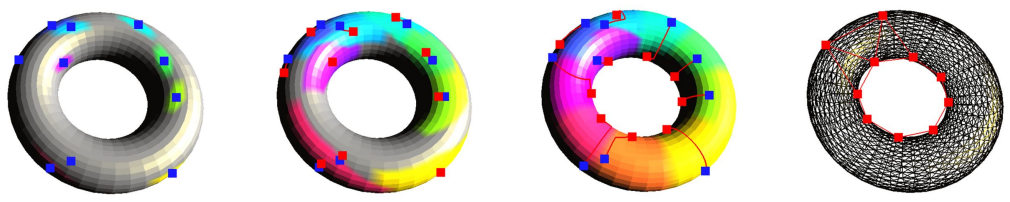

Fig. 3 Adaptive deployment of 10 robots (red) from start (blue), with isotropic curvature metric. The dual Delaunay graph at convergence is shown on the right.
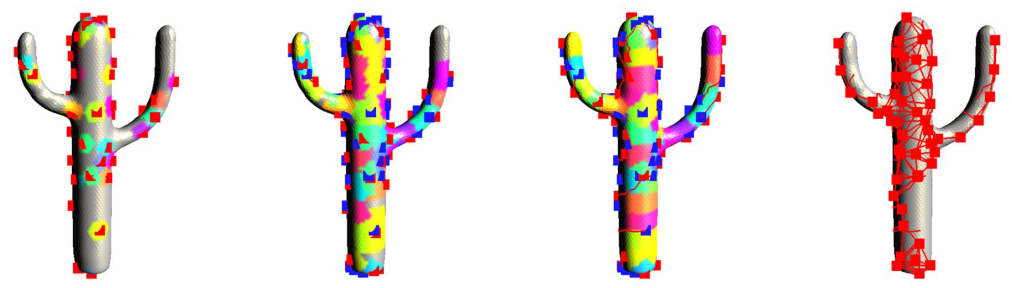

Fig. 4 Adaptive deployment of 50 robots (red) from start (blue), with anisotropic curvature metric. The dual Delaunay graph at convergence is shown on the right.

In the fourth experiment, the inner surface of an industrial tube structure is covered by five robots. Figure 6 shows one iteration of the hybrid area coverage method. The input is a $3 \mathrm{D}$ point cloud, which was recorded with a rotating Hokuyo URG-04LX laser scanner (Figure 6, left). From the point cloud, a triangle mesh is reconstructed. Five robots are deployed over the mesh in a first stage, using the second adaptive surface coverage algorithm (Algorithm 3) with $k=1$ rounds and uniform weights (Figure 6, center). In a second stage, each robot covers its Voronoi region with a sweeping motion (Figure 6, right). The path planner computes transferring as well as sweeping paths. Paths to transfer a robot to a goal position are planned by an $\mathrm{A}^{*}$ search, which generates a triangle strip path on the mesh. The sweeping paths to cover the surface area result from a covering strip planner, which is based on [16]. The robots are controlled by smooth vector fields generated along the strip, using concepts similar to [17]. Figure 5 on the right gives an example for a generated vector field that steers a robot toward the mass center of its Voronoi region.

\section{Conclusion}

In this paper, we have presented two adaptive surface coverage algorithms to deploy multiple robots on curved surfaces. The algorithms operate on triangle meshes and distribute the robots into discrete regions on the mesh. The size, positions and shapes of the regions are adaptive; they are influenced 

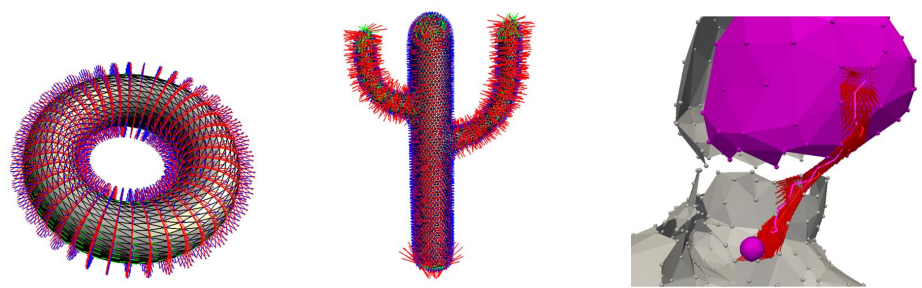

Fig. 5 Left, center: Principal curvature directions estimated for the torus and cactus models. Right: Close-up of the planned triangle strip path and the vector field generated along the strip for robot control toward the mass center of the Voronoi region.
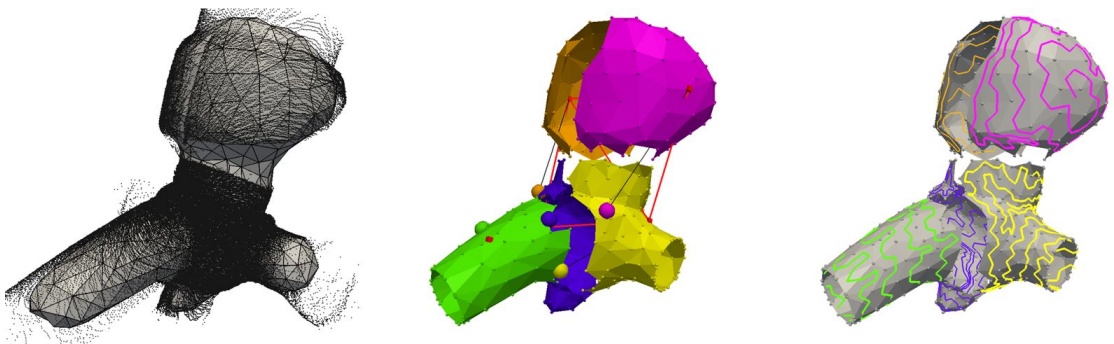

Fig. 6 Left: 3D laser point cloud and reconstructed triangle mesh of a steam chest tube. Center: Partition resulting from the deployment of five robots. Right: Covering triangle strip paths defining a sweeping pattern for each of the Voronoi regions.

by a metric tensor field defined on the surface. The adaptive surface coverage algorithms are applied under the hybrid coverage concept for surface area coverage. The algorithms implement the adaptive deployment of the robots. In a second stage, the robots cover the areas of their assigned regions by a sweeping motion. Depending on the environment and the coverage task, this procedure is iterated, which finally leads to complete coverage of the surface. The algorithms are successfully tested in several simulation experiments on different synthetic as well as more realistic mesh models.

In our future work, we plan to test the algorithms on real robots, with added localization and mapping functionalities. Regarding adaptive surface coverage, we will look at the optimization of pairwise partitions and the adaption of regions by user guidance. Concerning hybrid area coverage, it is interesting to further study schemes of robot relocation and redistribution of regions and tasks among robots, particularly for deployment, exploration and search scenarios in heterogeneous robot teams. 


\section{References}

1. J. Cortés, S. Martínez, T. Karatas and F. Bullo, "Coverage Control for Mobile Sensing Networks", in IEEE Transactions on Robotics and Automation, vol. 20, no. 2, pp. 243-255, 2004.

2. S. Meguerdichian, F. Koushanfar, M. Potkonjak and M. B. Srivastava, "Coverage Problems in Wireless Ad-hoc Sensor Networks", in Proc. of the 20th Annual Joint Conf. of the IEEE Computer and Communications Societies (INFOCOM), pp. 1380-1387, 2001.

3. M. Pavone, K. Savla and E. Frazzoli, "Sharing the Load", in IEEE Robotics and Automation Magazine, vol. 16, no. 2, pp. 52-61, 2009.

4. S. L. Smith, M. Schwager and D. Rus, "Persistent Robotic Tasks: Monitoring and Sweeping in Changing Environments", in IEEE Transactions on Robotics, vol. 28, no. 2, pp. 410-426, 2012.

5. P. N. Atkar, H. Choset, A. A. Rizzi and E. U. Acar, "Exact Cellular Decomposition of Closed Orientable Surfaces Embedded in $\mathfrak{R}^{3}$ ", in Proc. of the Int. Conf. on Robotics and Automation (ICRA), pp. 699-704, 2001.

6. A. Breitenmoser, F. Tâche, G. Caprari, R. Siegwart and R. Moser, "MagneBikeToward Multi Climbing Robots for Power Plant Inspection", in Proc. of the 9th Int. Conf. on Autonomous Agents and Multiagent Systems (AAMAS), 2010.

7. A. Breitenmoser, J.-C. Metzger, R. Siegwart and D. Rus, "Distributed Coverage Control on Surfaces in 3D Space", in Proc. of the IEEE/RSJ Int. Conf. on Intelligent Robots and Systems (IROS), pp. 5569-5576, 2010.

8. Q. Du and D. Wang, "Anisotropic Centroidal Voronoi Tessellations and Their Applications", in SIAM Journal on Scientific Computing, vol. 26, pp. 737-761, 2005.

9. S. Bhattacharya, Robert Ghrist and Vijay Kumar, "Multi-Robot Coverage and Exploration in Non-Euclidean Metric Spaces", in Proc. of the 10th Int. Workshop on the Algorithmic Foundations of Robotics (WAFR), 2012.

10. J. W. Durham, R. Carli, P. Frasca and F. Bullo, "Discrete Partitioning and Coverage Control for Gossiping Robots", in IEEE Transactions on Robotics, vol. 28 , no. 2 , pp. $364-378,2012$.

11. S. Yun and D. Rus, "Distributed Coverage with Mobile Robots on a Graph: Locational Optimization", in Proc. of the IEEE Int. Conf. on Robotics and Automation (ICRA), pp. 634-641, 2012.

12. S. Bougleux, G. Peyré and L. D. Cohen, "Anisotropic Geodesics for Perceptual Grouping and Domain Meshing", in Proc. of the 10th Europ. Conf. on Computer Vision (ECCV), pp. 129-142, 2008.

13. S. Valette, J.-M. Chassery and R. Prost, "Generic Remeshing of 3D Triangular Meshes with Metric-Dependent Discrete Voronoi Diagrams", in IEEE Transactions on Visualization and Computer Graphics, vol. 14, no. 2, pp. 369-381, 2008.

14. A. Gusrialdi, S. Hirche, T. Hatanaka and M. Fujita, "Voronoi Based Coverage Control with Anisotropic Sensors", in Proc. of the Am. Control Conf. (ACC), pp. 736-741, 2008.

15. M. Meyer, M. Desbrun, P. Schröder and A. H. Barr, "Discrete DifferentialGeometry Operators for Triangulated 2-Manifolds", in Proc. of VisMath, pp. 35-57, 2002.

16. M. Gopi, "Controllable Single-Strip Generation for Triangulated Surfaces", in Pacific Conf. on Computer Graphics and Applications, pp. 61-69, 2004.

17. G. A. S. Pereira, L. C. A. Pimenta, A. R. Fonseca, L. de Q. Corrêa, R. C. Mesquita, L. Chaimowicz, D. S. C. de Almeida and M. F. M. Campos, "Robot Navigation in Multi-Terrain Outdoor Environments", in Int. Journal of Robotic Research, vol. 28, no. 6, pp. 685-700, 2009. 